\title{
Bits-to-Symbol Mappings for Superposition Coding Based HARQ Systems
}

\author{
Chaitanya Tumula V. K. and Erik G. Larsson
}

\section{Linköping University Post Print}

\section{Tweet}

N.B.: When citing this work, cite the original article.

(C2013 IEEE. Personal use of this material is permitted. However, permission to reprint/republish this material for advertising or promotional purposes or for creating new collective works for resale or redistribution to servers or lists, or to reuse any copyrighted component of this work in other works must be obtained from the IEEE.

Original Publication:

Chaitanya Tumula V. K. and Erik G. Larsson, Bits-to-Symbol Mappings for Superposition Coding Based HARQ Systems, 2013, Proceedings of the IEEE Wireless Communications and Networking Conference (WCNC).

Pre-print available at: Linköping University Electronic Press http://urn.kb.se/resolve?urn=urn:nbn:se:liu:diva-87279 


\title{
Bits-to-Symbol Mappings for Superposition Coding Based HARQ Systems
}

\author{
Tumula V. K. Chaitanya and Erik G. Larsson
}

\begin{abstract}
We consider the mapping of bits-to-superimposed constellation symbols in terms of the achievable rate on the channel for the HARQ system using superposition coding as proposed in [2]. We show that using a Gray mapping of bits-tosuperimposed constellation symbols has better performance than the conventional natural mapping that results from superposition in signal space, for all the values of the superposition ratio. We also show through link-level simulations that the predicted gains in terms of achievable rate can be realized in practice using LDPC codes. Furthermore, we show that the optimal superposition ratio for the Gray mapping case results in conventional higher order constellation symbols after the superposition operation.
\end{abstract}

Index Terms-Superposition coding, bits-to-symbol mapping, hybrid ARQ.

\section{INTRODUCTION}

Superposition coding originally proposed in [1] for additive white Gaussian noise (AWGN) broadcast channels has found many applications recently in the wireless communications field. In many applications, superposition coding is used to utilize the resources more efficiently. Generally, superposition of two or more users data can be done in many ways, from superposition in signal (Euclidean) space as in [2], [3] to algebraic network coding as in [4]. In [2], a new hybridautomatic repeat request (HARQ) scheme was proposed based on superposition coding in the signal space. In this scheme, if a packet was received in error, it is retransmitted in the next timeslot superimposed on top of a new data packet. By contrast, in conventional retransmission schemes, an erroneous packet is transmitted alone in the next timeslot. The advantage of superposition of a new data packet along with an erroneous previously transmitted data packet is that the resources are utilized in a more efficient manner. However the superposition operation also introduces inter-packet-interference (IPI). Even with IPI, this new retransmission scheme was shown to perform better in terms of link-layer throughput than a conventional HARQ scheme.

Related Work and Contributions: Previously, bits-tosymbol mappings have been extensively studied for different scenarios: for trellis-coded modulation in [5], for bitinterleaved coded modulation (BICM) with iterative decoding in [6] and for multiple packet transmissions in [7]. As for our knowledge, none of the previous works addresses the bits-to-symbol mappings when multiple packets of data are transmitted together using superposition coding in the context

The authors are with the Dept. of Electrical Engineering (ISY), Linköping University, Linköping, Sweden. E-mail: \{tvk,egl $\} @$ isy.liu.se. This work was supported in part by Ericsson, VINNOVA, the Swedish Foundation for Strategic Research (SSF) and ELLIIT.

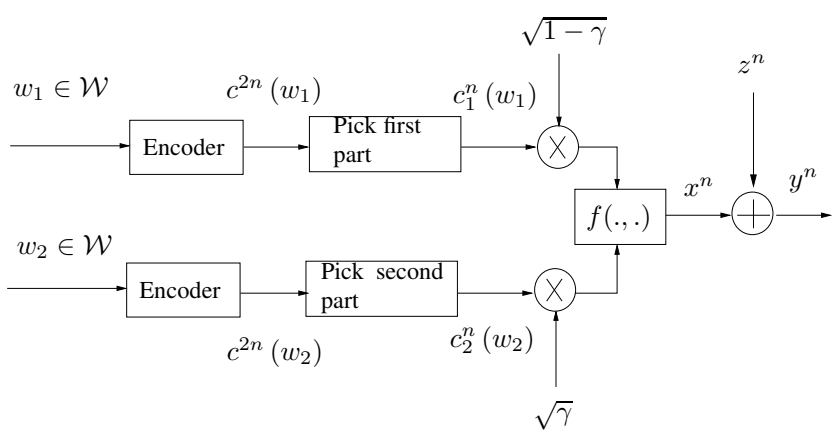

Figure 1. System model for HARQ system using superposition coding.

of a HARQ system. Hence in this work, we consider an incremental redundancy based HARQ system using superposition coding as in [2]. Instead of using a linear mapping from the modulation symbols of the individual data packets to the superimposed constellation symbols (this results in the natural labeling of the bits in the superimposed constellation) as done in [2], we consider a non-linear mapping which results in a Gray mapping of bits from from the original data packets to the superimposed constellation symbols.

We compare these two bits-to-superimposed constellation symbol mappings in terms of the achievable rate on an additive white Gaussian noise (AWGN) channel. We show that using a non-linear mapping which results in a Gray mapping of bitsto-superimposed constellation symbols provides a considerable gain in terms of the achievable rate for the HARQ system with superposition coding relative to the original technique in [2]. We also show that the predicted gains for the achievable rate can be realized in practice using LDPC codes.

\section{System MODEL}

In this section, we describe the system model for the HARQ system using superposition coding. We consider a point-to-point communication link with HARQ, in which an erroneous message is retransmitted along with a new message using superposition coding [2]. We consider a HARQ scheme which allows only one additional retransmission for an erroneously received data packet and also assume that all the transmitted messages utilize one additional retransmission as in [2]. The system model is shown in Fig. 1, where $w_{1}, w_{2} \in \mathcal{W}=\left\{1,2, \cdots 2^{\lceil 2 n R\rceil}\right\}$ denote a new message and a retransmitted message which was received in error, respectively. These messages are encoded by an encoder $\left(\varphi: \mathcal{W} \rightarrow \mathcal{S}_{1}^{n} \times \mathcal{S}_{2}^{n}\right)$ to obtain the codewords $c^{2 n}\left(w_{1}\right), c^{2 n}\left(w_{2}\right) \in \mathcal{C}$. The set $\mathcal{C}=\left\{c^{2 n}(w)\right\}_{w=1}^{2^{\lceil 2 n R\rceil}}=$ $\left\{c^{2 n}(1), c^{2 n}(2), \cdots, c^{2 n}\left(2^{\lceil 2 n R\rceil}\right)\right\}$ denotes the codebook 


\begin{tabular}{cccc}
$s_{1}$ & $s_{2}$ & $s_{3}$ & $s_{4}$ \\
\hline$* 0$ & $*$ & $*$ & $*$
\end{tabular}

(a) Natural mapping of bits-to-superimposed symbols for the signal space superposition of BPSK constellations.

$\begin{array}{cccc}s_{1} & s_{2} & s_{3} & s_{4} \\ * * & * & *\end{array}$

(b) One possible Gray mapping for superposition of BPSK constellations.

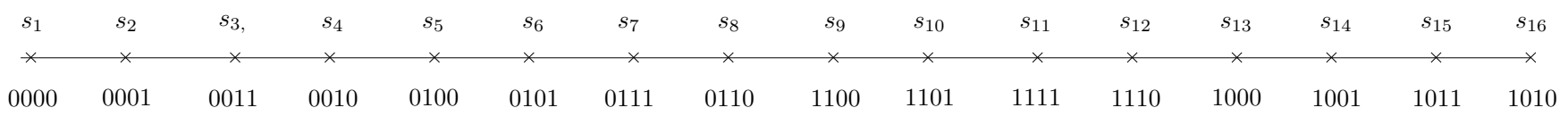

(c) Natural mapping of bits-to-superimposed symbols for the signal space superposition of 4-PAM constellations.

$\begin{array}{cccccccccccccccc}s_{1} & s_{2} & s_{3} & s_{4} & s_{5} & s_{6} & s_{7} & s_{8} & s_{9} & s_{10} & s_{11} & s_{12} & s_{13} & s_{14} & s_{15} & s_{16} \\ \star & \times & \times & \times & \times & \times & \times & \times & \times & \times & \times & \times & \times & \times & \times & \times \\ 0000 & 0001 & 0011 & 0010 & 0110 & 0111 & 0101 & 0100 & 1100 & 1101 & 1111 & 1110 & 1010 & 1011 & 1001 & 1000\end{array}$

(d) One possible Gray mapping for superposition of 4-PAM constellations.

Figure 2. Two different bits-to-symbol mappings for superposition coding of two packets.

and each codeword has length $2 n$. A codeword corresponding to a given message $i$ consists of two parts, $c^{2 n}(i)=$ $\left[c_{1}^{n}(i) c_{2}^{n}(i)\right], \forall 1 \leq i \leq 2^{\lceil 2 n R\rceil}$, where $c_{1}^{n}(i), c_{2}^{n}(i)$ denote the first and the second part of the codeword $c^{2 n}(i)$ respectively. We assume that the entries of all the codewords in $\mathcal{C}$ come from a finite-size PAM constellation. ${ }^{1}$ Specifically, we assume that BPSK or 4-PAM modulation per real dimension is used for the data packets (before the superposition operation). ${ }^{2}$ We also assume that the components of the codewords take on the signal points in the constellation with equal probability.

During the transmission, the transmitter picks the first part of the codeword corresponding to a new message and the second part of the codeword corresponding to a retransmitted message. The codeword parts corresponding to a new message and a retransmitted message are multiplied with scalar constants $\sqrt{1-\gamma}$ and $\sqrt{\gamma}$ respectively, where $\gamma$ (superposition ratio) denotes the fraction of power used for the codeword part corresponding to a retransmitted message. A mapping function $f(.,$.$) operates on the codeword parts (corresponding$ to a new message and a retransmitted message) to from $x^{n}$, which will be sent on the channel in $n$ channel uses. The mapping function operates on the components of codewords independently. For the signal space superposition considered in [2], $f(.,$.$) is a simple algebraic addition operation. Assuming$ BPSK and 4-PAM modulation for the codeword parts, and with $f(.,$.$) being algebraic addition operation, we obtain the$ superimposed constellation symbols defined by:

$$
S_{4} \triangleq\left\{s_{1} \triangleq-\sqrt{1-\gamma}-\sqrt{\gamma}, s_{2} \triangleq-\sqrt{1-\gamma}+\sqrt{\gamma},\right.
$$

${ }^{1}$ Even though, we assumed that the entries of the first and the second parts of the codeword come from the same finite size PAM constellation, one can also think of superposition coding based HARQ scheme for other possible scenarios as well. For example, the entries of the first part of codeword can come 4-PAM constellation and the components of the second part can come from BPSK constellation. With this model, in each time slot, retransmitted data packet consisting of BPSK modulation symbols is superimposed on an original data packet consisting of 4-PAM modulation symbols. The superimposed constellation in this case consists of 8 signal points.

${ }^{2}$ This idea can be extended to 2-dimensional constellations like M-QAM and M-PSK.

$$
\begin{gathered}
\left.s_{3} \triangleq \sqrt{1-\gamma}-\sqrt{\gamma}, s_{4} \triangleq \sqrt{1-\gamma}+\sqrt{\gamma}\right\}, \text { and } \\
S_{16} \triangleq\left\{s_{1} \triangleq-\sqrt{1-\gamma} x_{1}-\sqrt{\gamma} x_{1}, s_{2} \triangleq-\sqrt{1-\gamma} x_{1}-\sqrt{\gamma} x_{2}\right. \\
\left., \cdots, s_{16} \triangleq \sqrt{1-\gamma} x_{1}+\sqrt{\gamma} x_{1}\right\}
\end{gathered}
$$

respectively, where $x_{1}=\sqrt{0.8}+\sqrt{0.2}$ and $x_{2}=\sqrt{0.8}-\sqrt{0.2}$. Note that the algebraic addition of the codeword components results in the conventional natural mapping of bits-tosuperimposed symbols as shown in Figs. 2(a) and 2(c).

In order to look at the other mapping functions $f(.,$. which can result in a better performance of the superposition coded HARQ scheme, we consider all possible mappings from the modulation symbols (bits) of the data packets to the superimposed symbols in (1). In the following, we first assume BPSK for the modulation of packets before the superposition operation. Our objective is to find an optimal mapping of bits $\left(b_{1} b_{2}\right) \in\{00, \cdots, 11\}$ onto the modulation symbols of $S_{4}$ in terms of maximizing the achievable rate on the channel. ${ }^{3}$ In this case, there are a total of $4 !=24$ possible mappings of which 12 are redundant (replacing bit ' 1 ' with bit ' 0 ' and vice versa) in terms of mutual information. The set of all possible mappings for $\left(s_{1}, s_{2}, s_{3}, s_{4}\right)$ to be considered are:

$$
\begin{array}{r}
\text { (I) }(00,01,10,11) ;(\mathrm{II})(00,01,11,10) ;(\mathrm{III})(00,10,01,11) \\
(\mathrm{IV})(00,10,11,01) ;(\mathrm{V})(00,11,01,10) ;(\mathrm{VI})(00,11,10,01) ; \\
\text { (VII) }(01,00,10,11) ;(\mathrm{VIII})(01,00,11,10) ;(\mathrm{IX})(01,10,00,11) ; \\
(\mathrm{X})(01,10,11,00) ;(\mathrm{XI})(01,11,00,10) ;(\mathrm{XII})(01,11,10,00) ;
\end{array}
$$

Mapping (I) corresponds to the conventional natural mapping. Any mapping other than the mapping (I) for the superimposed symbols can be seen as a non-linear mapping $f(.,$.$) from$ the modulation symbols (bits) in the original data packets to the superimposed symbols. For a given mapping, let $d=\left(d_{1}, d_{2}, d_{3}\right)$ denote the number of bit position changes from one symbol to the next symbol in the superimposed

\footnotetext{
${ }^{3}$ In this work, we are not interested in finding the optimal encoding and decoding strategies. We assume that we have a channel code which works for the scenario described in Section II.
} 
constellation. The mappings (II), (IV), (VII) and (XII) denote the possible Gray mappings with $d=(1,1,1)$ and they are equivalent in the sense of mutual information (to be precise, they have the same $R(\gamma)$ as defined in Section III). Similarly, the mappings (I), (III), (VIII) and (XI) are equivalent with $d=(1,2,1)$ and so are the mappings $(\mathrm{V}),(\mathrm{VI}),(\mathrm{IX})$ and $(\mathrm{X})$ with $d=(2,1,2)$.

With the superposition of 4-PAM constellations, there are in total $\frac{16 !}{2}$ different mappings for $\left(b_{1} b_{2} b_{3} b_{4}\right) \in$ $\{0000, \cdots, 1111\}$ onto the symbols in $S_{16}$. Since it is mathematically intractable to group these combinations in terms of bit position changes from one symbol to the next symbol, and it has also been verified that the performance of the mapping group with $d=(2,1,2)$ is inferior to the performance of the other two mapping groups for the BPSK superposition case, we consider only two bits-to-superimposed constellation symbol mappings in this study: The natural mapping as shown in Figs. 2(a) and 2(c) and the Gray mapping as shown in Figs. 2(b) and 2(d). Note that $\gamma=\frac{1}{5}$ for BPSK and $\gamma=\frac{1}{17}$ for 4-PAM results in uniform 4-PAM and uniform 16-PAM constellations after the superposition operation in Fig. 2.

We study the performance of these two bits-to-superimposed symbol mappings in terms of the achievable rate. Assuming an AWGN channel as in Fig. 1, the received signal at the destination in each channel use can be written as (we drop the channel use index for notational convenience):

$$
y=x+z,
$$

where $x \in S_{4}$ and $z$ denotes an AWGN sample with $z \sim$ $\mathcal{C N}\left(0, \sigma_{d}^{2}\right)$. Note that $\mathbb{E}\left|x^{2}\right|=1$ by design. We define the average signal-to-noise ratio (SNR) as $\mathrm{SNR} \triangleq \frac{\mathbb{E}\left|x^{2}\right|}{\sigma_{d}^{2}}=\frac{1}{\sigma_{d}^{2}}$.

On the receiver side, when demodulating the symbols, we treat the symbols corresponding to a retransmitted message as interference (nuisance) for which no side information is available. While obtaining the soft information for the bits corresponding to the erroneous packet, we do not use soft decoder output from the previous timeslot to aid the demodulation during the current timeslot. One could however think of iterative-detection based schemes where the soft information from the previous data blocks are used to aid the demodulation during the current timeslot.

\section{Achievable Rate Analysis}

In this section, we derive expressions for the achievable rate on the channel both for the natural mapping and the Gray mapping of bits-to-superimposed symbols for the superposition coding based HARQ system described in Section II.

Assuming BPSK modulated data packets, we compute the total mutual information in terms of bits per channel use (bpcu) at the destination after the allowed additional retransmission. ${ }^{5}$ The achievable rate on the channel after two timeslots is the

\footnotetext{
${ }^{4}$ We use Gray mapping of the bits onto the 4-PAM constellation before the superposition operation to obtain the superimposed 16-PAM constellation.

${ }^{5}$ When we refer to bpcu values in this work, they correspond to bits per channel use per real dimension.
}

same as the total mutual information of the two bits (corresponding to the new data packet bit and the retransmitted data packet bit) sent on the channel in two successive timeslots. We can express it in terms of bpcu as:

$$
R(\gamma)=\frac{1}{2}\left[I_{\gamma}\left(y ; b_{1}(x)\right)+I_{\gamma}\left(y ; b_{2}(x)\right)\right],
$$

where $x \in S_{4} . I_{\gamma}\left(y ; b_{1}(x)\right)$ and $I_{\gamma}\left(y ; b_{2}(x)\right)$ are the mutual information values at the destination during two successive timeslots corresponding to a given message. The maximum achievable rate on the channel can be written as:

$$
R^{*}=\max _{\gamma} R(\gamma)
$$

For notational convenience, we denote the bits of a superimposed constellation symbol as $b_{k}$ instead of $b_{k}(x)$. Since the bit $b_{k}$ is equally likely to be 0 or 1 , for $k=1$ and 2 , we can write:

$I_{\gamma}\left(y ; b_{k}\right)=\frac{1}{2}\left[\int \sum_{m=0}^{1} f\left(y \mid b_{k}=m\right) \log _{2}\left(\frac{f\left(y \mid b_{k}=m\right)}{f(y)}\right) d y\right]$

Now writing the conditional distributions for the received signal, for the natural mapping case, we have:

$$
\begin{aligned}
& f\left(y \mid b_{1}=0\right)=\left(f\left(y \mid s_{1}\right)+f\left(y \mid s_{2}\right)\right) / 2 \\
& f\left(y \mid b_{1}=1\right)=\left(f\left(y \mid s_{3}\right)+f\left(y \mid s_{4}\right)\right) / 2 \\
& f\left(y \mid b_{2}=0\right)=\left(f\left(y \mid s_{1}\right)+f\left(y \mid s_{3}\right)\right) / 2 \\
& f\left(y \mid b_{2}=1\right)=\left(f\left(y \mid s_{2}\right)+f\left(y \mid s_{4}\right)\right) / 2
\end{aligned}
$$

and for the Gray mapping case, we have:

$$
\begin{aligned}
& f\left(y \mid b_{1}=0\right)=\left(f\left(y \mid s_{1}\right)+f\left(y \mid s_{2}\right)\right) / 2 \\
& f\left(y \mid b_{1}=1\right)=\left(f\left(y \mid s_{3}\right)+f\left(y \mid s_{4}\right)\right) / 2 \\
& f\left(y \mid b_{2}=0\right)=\left(f\left(y \mid s_{1}\right)+f\left(y \mid s_{4}\right)\right) / 2 \\
& f\left(y \mid b_{2}=1\right)=\left(f\left(y \mid s_{2}\right)+f\left(y \mid s_{3}\right)\right) / 2
\end{aligned}
$$

Also,

$$
\begin{aligned}
f(y) & =\frac{1}{4} \sum_{l=1}^{4} f\left(y \mid s_{l}\right) \\
f\left(y \mid s_{l}\right) & =\frac{1}{\sqrt{2 \pi \sigma_{d}^{2}}} \exp \left(\frac{-\left(y-s_{l}\right)^{2}}{2 \sigma_{d}^{2}}\right) \text { for } l \in\{1,2,3,4\}
\end{aligned}
$$

As we can see from (6) and (7), $f\left(y \mid b_{1}=m\right)$ for $m=0$ and 1 is the same for both the natural mapping and the Gray mapping. Hence, we have the same $I_{\gamma}\left(y ; b_{1}\right)$ for both the mappings. Note that the distributions in (6), (7) and (8) are Gaussian mixture distributions. An analysis can be done for other modulation schemes as well by writing similar expressions for the conditional probabilities. In particular, similar expressions can be written out for the case of 4-PAM baseline modulation, but we omit that here due to space limitations. 


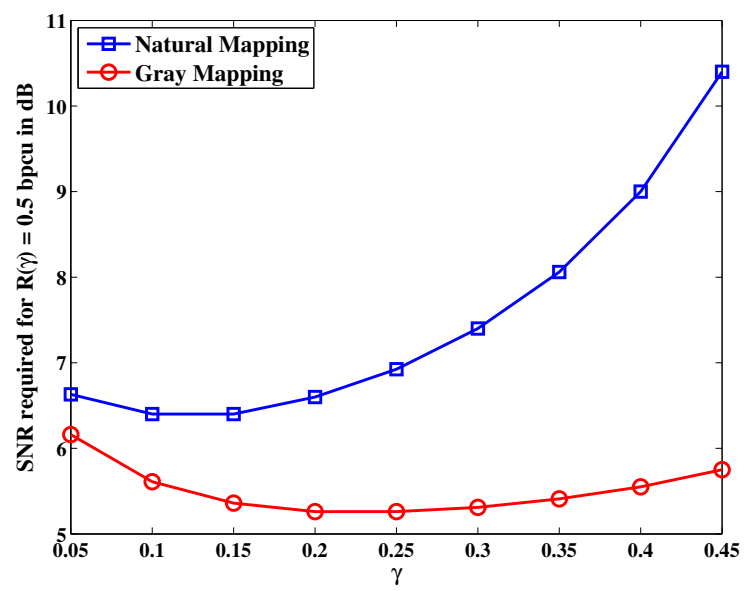

(a) SNR required to achieve a rate of $0.5 \mathrm{bpcu}$.

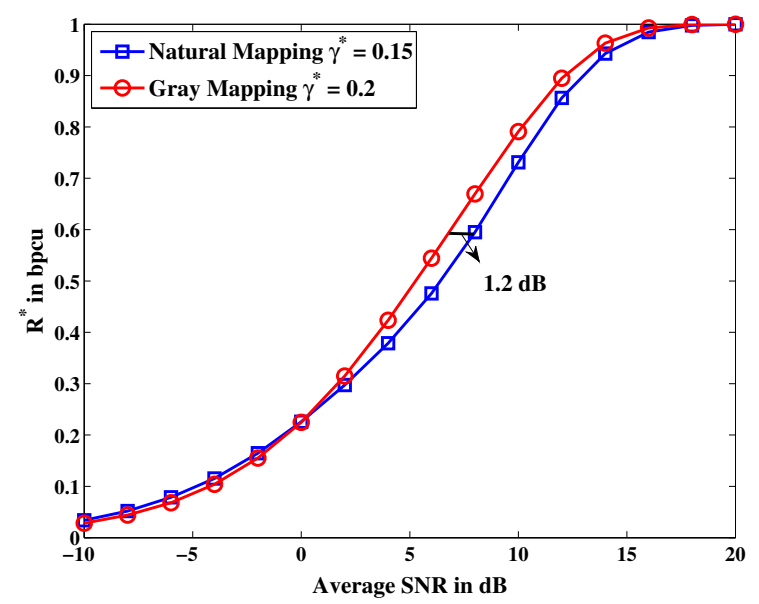

(b) Maximum achievable rate as a function of SNR.

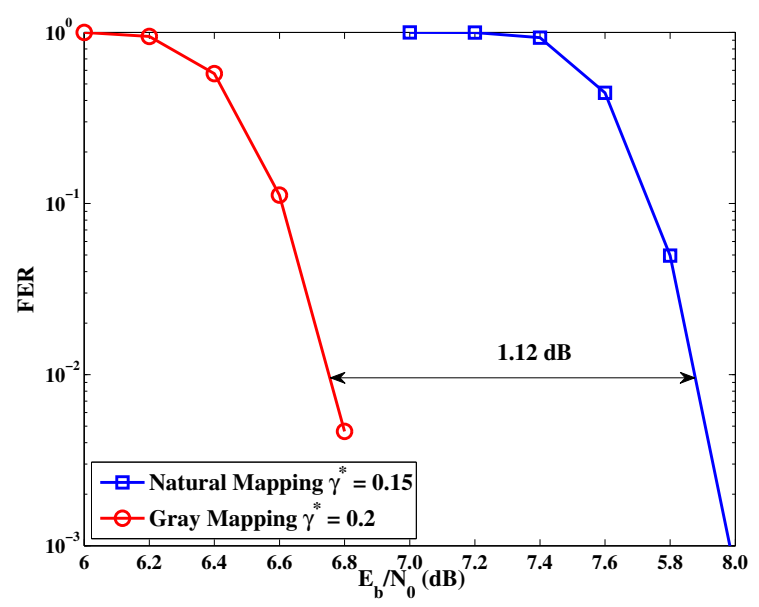

(c) Empirical FER performance comparison using a rate-1/2 LDPC code.

Figure 3. Plot showing the performance comparison of natural mapping with the Gray mapping for the superposition of BPSK constellations.

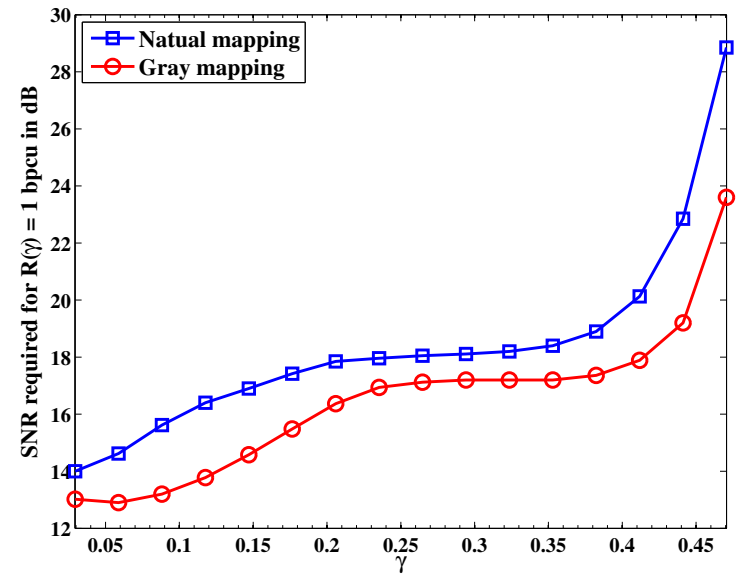

(a) SNR required to achieve a rate of 1 bpcu.

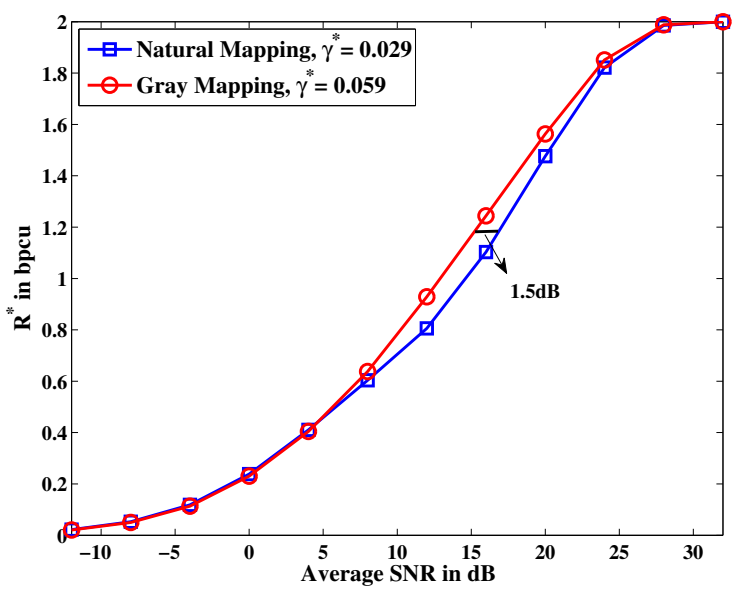

(b) Maximum achievable rate as a function of SNR.

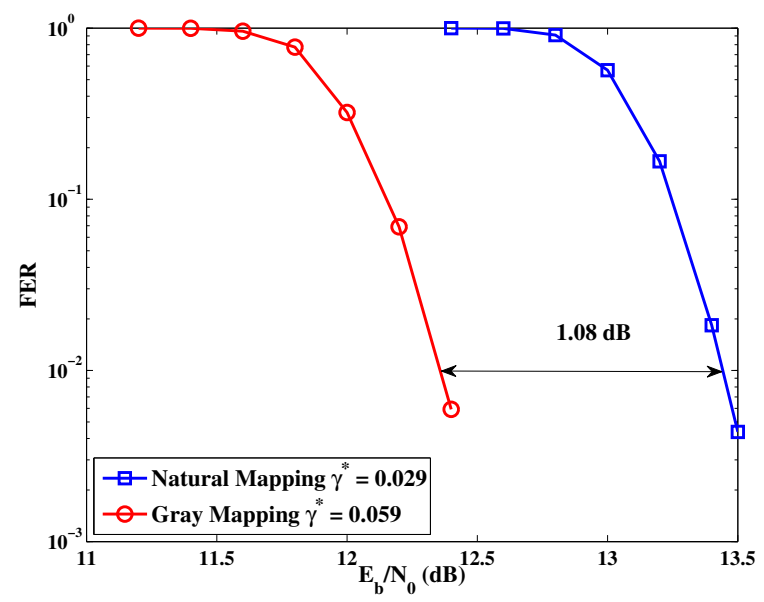

(c) Empirical FER performance comparison using a rate-1/2 LDPC code.

Figure 4. Similar to Fig. 3 but for the superposition of 4-PAM constellations. 


\section{RESUlts}

In this section, we present numerical results for the HARQ system using superposition coding described in Section II. Since the mutual information expressions presented in this work involve Gaussian mixture distributions, we used MonteCarlo integration to obtain the results.

Fig. 3 shows the results for the superposition of BPSK constellations. In Fig. 3(a), we plot average SNR in $\mathrm{dB}$ required for an achievable rate of $0.5 \mathrm{bpcu}$ both for the natural mapping and the Gray mapping cases. From the Fig. 3 (a), we see that, for all the values of superposition ratio $0.05 \leq \gamma \leq 0.45$, Gray mapping has better performance than the natural mapping. The optimal choice of $\gamma$ for the natural mapping and the Gray mapping turned out to be 0.15 and 0.2 respectively, and the gain of using Gray mapping over natural mapping for optimal $\gamma$ is $1.2 \mathrm{~dB}$. We can also observe from Figure 3(a) that as $\gamma$ approaches 0.5 , the average SNR required for $R(\gamma)$ to reach $0.5 \mathrm{bpcu}$ rapidly increases for the natural mapping case. This can be explained by considering Fig. 2(a), from which we see that when $\gamma=0.5$, the mutual information that can be obtained each with bits $b_{1}$ and $b_{2}$ is upper bounded by 0.5 bpcu. By contrast, with the Gray mapping (see Fig 2(b)), we can still obtain a mutual information of $0.5 \mathrm{bpcu}$ and 1 bpcu each with bits $b_{1}$ and $b_{2}$ respectively (for the Gray mapping case, even when $\gamma=0.5$, one can decode the bit $b_{2}$ without any uncertainty).

Fig. 3(b) shows the result for the maximum achievable rate $R^{*}$ as a function of the average SNR for both the natural mapping and the Gray mapping cases. Fig. 3(c) shows a simulated link-level performance comparison of the Gray mapping and the natural mapping using a systematic $(3,6)$ LDPC code (rate 1/2) with a block size of 10000 bits. $^{6}$ During the first transmission, we transmit the systematic part of the codeword and during the retransmission phase, we transmit only the parity bits in an incremental redundancy fashion. We used BPSK modulation before the superposition operation. Note that this corresponds to a spectral efficiency of $0.5 \mathrm{bpcu}$. As we can can see from the figure, at a frame-error-rate (FER) of $10^{-2}$, Gray mapping with optimal superposition ratio has a performance gain of $1.12 \mathrm{~dB}$ over the natural mapping with optimal superposition ratio. This empirically observed gain matches very well with the prediction from the mutual information analysis.

For the superposition of 4-PAM constellations, the results are shown in Fig. 4. In Fig. 4(a), we compare the average SNR required in $\mathrm{dB}$ to attain an achievable rate of $1 \mathrm{bpcu}$ for both the natural mapping and the Gray mapping. We can observe from the figure that, as $\gamma$ approaches 0.5 , the required average SNR value increases both for the natural mapping and the Gray mapping. For the 4-PAM case, the optimal superposition ratio value for the natural mapping and the Gray mapping case is 0.029 and 0.059 respectively. The

\footnotetext{
${ }^{6} \mathrm{We}$ choose these parameters for our simulation as it is well known that LPDC codes with very large block sizes can operate close to theoretical Shannon capacity limit [8].
}

gain for the Gray mapping case with optimal $\gamma$ is $1.1 \mathrm{~dB}$ over the natural mapping case with optimal $\gamma$. Figs. 4(b) and 4(c) show the maximum achievable rate and the link-level FER performance for the natural mapping and the Gray mapping with optimum superposition ratio values. We can see from 4(c) that the predicted gain of $1.1 \mathrm{~dB}$ at $1 \mathrm{bpcu}$ for the Gray mapping can be realized in practice with an LDPC code. ${ }^{7}$

\section{CONClusions And Future Work}

We considered the mapping of bits-to-superimposed constellation symbols for a HARQ system using superposition coding of the type proposed in [2]. The use of Gray mapping of bits-to-superimposed symbols provides a considerable gain over the natural mapping case in terms of the achievable rate. We also demonstrated through link-level simulation results that the predicted gains can be realized in practice using LDPC codes. The optimal superposition ratio $\gamma^{*}$ for a HARQ system using BPSK modulation and Gray mapping of bitsto-superimposed symbols is 0.2 . This value of $\gamma^{*}$ results in uniform 4-PAM constellation points for the superimposed constellation symbols. Similarly, a 4-PAM modulated HARQ system using superposition coding with one additional retransmission has an optimal superposition ratio of $\gamma^{*}=0.059$ and this value results in uniform 16-PAM constellation points for the superimposed constellation.

In our present work, the receiver did not use iterative decoding between different parts of the codeword received in different time slots. We plan to extend the optimization of bits-to-superimposed symbol mappings for the case of iterative decoding at the receiver, and this may potentially lead to different optimal mappings.

\section{REFERENCES}

[1] T. M. Cover, "Broadcast channels," IEEE Trans. Info. Theory, vol. 18, no. 1, pp. 2-14, Jan. 1972.

[2] R. Zhang and L. Hanzo, "Superposition-coding-aided multiplexed hybrid ARQ scheme for improved end-to-end transmission efficiency," IEEE Trans. Vech. Tech., vol. 58. no. 8, pp. 4681-4686, Oct. 2009.

[3] F. Takahashi and K. Higuchi, "HARQ for predetermined-rate multicast channel," Proc. of IEEE VTC, Taipei, Taiwan, pp. 1-5, May 2010.

[4] L. Xiao, T. E. Fuja, J. Kliewer, and D. J. Costello, "A network coding approach to cooperative diversity," IEEE Trans. Inf. Theory, vol. 53, no. 10, pp. 3714-3722, Oct. 2007.

[5] G. Ungerboeck, "Channel coding with multilevel/phase signals," IEEE Trans. Inf. Theory, vol. 28, no. 1, pp. 55-67, Jan. 1982.

[6] F. Schreckenbach, N. Görtz, J. Hagenauer, and G. Bauch, "Optimization of symbol mappings for bit-interleaved coded modulation with iterative decoding," IEEE Comm. Letters, vol. 7, no. 12, pp. 593-595, Dec. 2003.

[7] H. Samra, Z. Ding, and P. M. Hahn, "Symbol mapping diversity design for multiple packet transmissions," IEEE Trans. Commun., vol. 53, no. 5, pp. 810-817, May 2005.

[8] T. J. Richardson, M. A. Shokrollahi, and R. L. Urbanke,. "Design of capacity-approaching irregular low-density parity-check codes," IEEE Trans. on Inf. Theory, vol.47, no.2, pp. 619-637, Feb. 2001.

\footnotetext{
${ }^{7}$ The same systematic $(3,6)$ - LDPC code with a block size of 10000 bits used for the simulation
} 